\title{
MEDICIÓN DE LAS FUERZAS DE CORTE CON UN DINAMÓMETRO PIEZO ELÉCTRICO DURANTE EL TALADRADO TÉRMICO POR FLUENCIA ${ }^{1}$
}

\author{
JUAN GODOY R. ${ }^{2}$ JORGE VERGARA D. ${ }^{2}$ JAIME VILLANUEVA A. ${ }^{2}$ \\ MARTÍN QUISPE Y. ${ }^{2} \quad$ PERCY OVIEDO O. $^{2} \quad$ FRANZ TIKAL $^{3} \quad$ STEFAN DAMM $^{3}$ \\ Recibido el 13 de junio de 2003, aceptado el 19 de agosto de 2003
}

\begin{abstract}
RESUMEN
A través de una cooperación internacional con la Universidad de Kassel de Alemania, se desarrolló un proyecto conjunto para determinar las fuerzas de avance y momento torsor en chapas de cobre y latón en el proceso de taladrado por fluencia térmica (TFT). En el Laboratorio de Procesos de Manufactura de la Universidad de Tarapacá fue instalado un equipo para medir fuerzas y momentos, conectado a un PC mediante una tarjeta de adquisición de datos. Mediante el software XACT, se obtuvieron las curvas de la fuerza de avance Fz y el momento torsor Mz, generadas durante el proceso.
\end{abstract}

Inicialmente con masas patrones y una llave de torque, se calibró el dinamómetro. Posteriormente se hicieron mediciones en las láminas de cobre y latón. Se concluyó que las fuerzas de avance y momentos torsores son mayores en chapas de latón cuando son comparadas con las chapas de cobre, para un mismo espesor.

Palabras Claves: Procesos de manufactura, mediciones, taladrado no convencional, láminas de cobre

\begin{abstract}
Through an international cooperation with the University of Kassel, Germany a research project was developed for studying the cutting forces components and momentum determination in thin Copper and Brass sheets during the Thermal Flow Drilling (TFD) processes. In the Manufacturing Processes Laboratory of the Mechanicals Emgineering Department of Universidad de Tarapacá, Arica, Chile, equipment for measuring forces and torque measurements was installed. It was connected to a data card and installed in a pc bus. With the AXACT software it was possible to develop the feed force component and momentum or torque curves.
\end{abstract}

Initially, with standard mass and a torque wrench the equipment was calibrated. After this, feed force and torque measurements were made in those thin Copper and Brass sheets. As a conclusion the feed force and the torque momentum were bigger for Brass than Copper sheets of the same thickness.

Keywords: Manufacture process, measurements, non-conventional drilling, copper sheet

\section{INTRODUCCIÓN}

Es indispensable conocer las fuerzas de corte y el torque que produce una herramienta de corte cuando mecaniza un material, para la selección adecuada de la máquina herramienta. Para el caso del taladrado por fluencia térmica (TFT) o "Thermal Flow Drilling (TFD)", la información existente relacionada con los datos de corte, está referida principalmente a aceros y aleaciones de aluminio. La información sobre parámetros de corte en cobre y sus aleaciones es muy escasa en la literatura y se refieren principalmente al proceso convencional por arranque de viruta [1], [2].

El proceso TFT, es un proceso de mecanizado sin arranque de viruta que en los últimos años ha adquirido relevancia industrial, debido a los avances tecnológicos en el área de la Ciencia de los Materiales [3].

Es conveniente contar con datos de corte específicos sobre este nuevo proceso, porque las herramientas existentes han sido fabricadas para el mecanizado

\footnotetext{
1 Universidad de Tarapacá, Laboratorio de Procesos de Manufactura, Dpto. de Mecánica, Arica - Chile

2 Universidad de Tarapacá, Dpto. Mecánica, Casilla 6-D, Arica-Chile,jmgodoy@uta.cl, jvergara@uta.cl, jvillanu@uta.cl

3 Universidad de Kassel, Instituto de Producción y Logística, Alemania
} 
convencional con remoción de viruta, considerando su uso masivo en acero y sus aleaciones. En este nuevo proceso, los datos existentes también están dirigidos al acero y sus aleaciones, pero los nuevos datos permitirían optimizar los parámetros operacionales de mecanizado, evaluar y rediseñar las herramientas de corte, en la búsqueda de nuevas utilizaciones para el cobre y sus aleaciones.

El proceso TFT puede ser dividido en cuatro etapas que son vistas en la Fig. 1.

Fase inicial: En esta etapa, la fuerza de avance es creciente hasta alcanzar un valor máximo. En esa etapa el momento torsor es pequeño. La herramienta está lista para taladrar la chapa.

Fase de Fluencia: En esta etapa, el material fluye en forma axial y radial, la fuerza de avance disminuye y el momento torsor crece hasta alcanzar un máximo valor. La temperatura del material alcanza un máximo en esta fase [4], [5]. La herramienta taladra la chapa, el material fluye.

Fase de acabado: En esta fase, normalmente se aplasta la rebaba superior, corona, alcanzando un máximo en la fuerza de avance y momento torsor. La herramienta termina su curso, en algunas aplicaciones, normalmente la rebaba superior es eliminada.

Fase Final: La herramienta es retirada de la chapa.

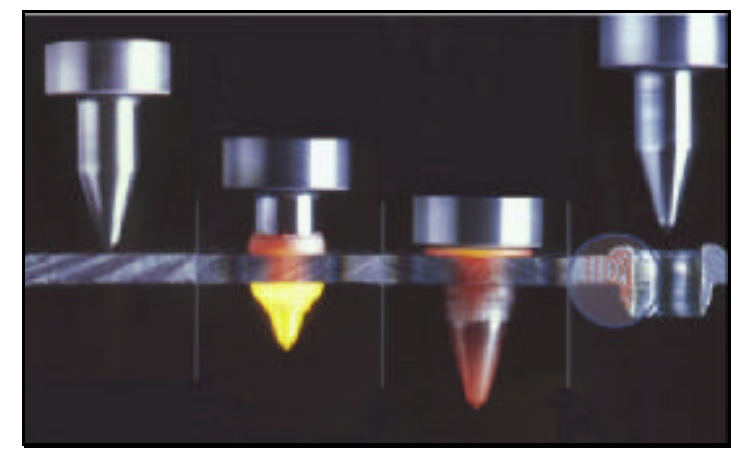

Fig. 1.- Etapas del proceso de taladrado térmico por fluencia.

\section{EQUIPOUTILIZADO}

En la Fig. 2 se muestra el esquema de adquisición de datos del Centro de Mecanizado HAAS. Los cuerpos de prueba a ser maquinados son fijados a un dispositivo instalado sobre el dinamómetro piezoeléctrico KISTLER.

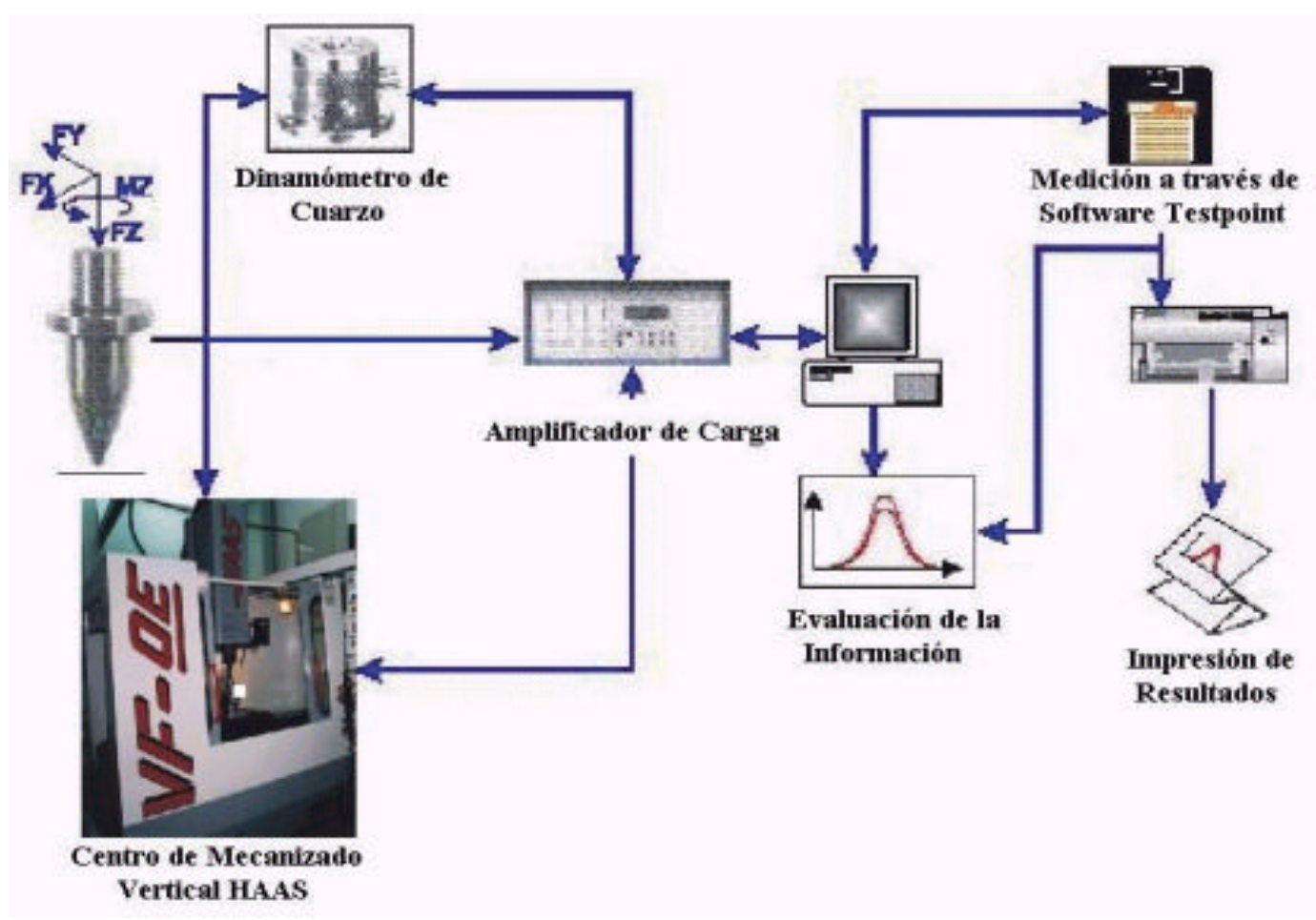

Fig. 2.- Esquema de adquisición de datos 
Cuando la herramienta inicia el mecanizado, el dinamómetro detecta la fuerza aplicada en sus tres componentes, además del torque. Las señales eléctricas emitidas por el dinamómetro llegan al amplificador de carga del sistema, aquí las señales son adaptadas para que ingresen a una tarjeta de adquisiciones de datos instalada en el bus de un PC, donde son procesados mediante el software TESTPOINT. En el PC los datos son graficados mediante el software XACT.

El proceso de calibración del dinamómetro fue realizado de acuerdo a la Fig. 3 y los resultados de la calibración pueden ser observados en la Fig. 4. Para la calibración de la fuerza de avance, se instaló sobre el dinamómetro una masa de $10 \mathrm{Kg}$ [98.1 N]. Para la calibración del momento, se empleó una llave de torque y se aplicó un momento de $35 \mathrm{Kg}$-m [343,35 Nm]. Con la aplicación de la masa y el torque, se realizaron lecturas de fuerza y momento que se regularon directamente en el amplificador de carga, hasta alcanzar los valores patrones mediante el software TESTPOINT, Fig. 4.

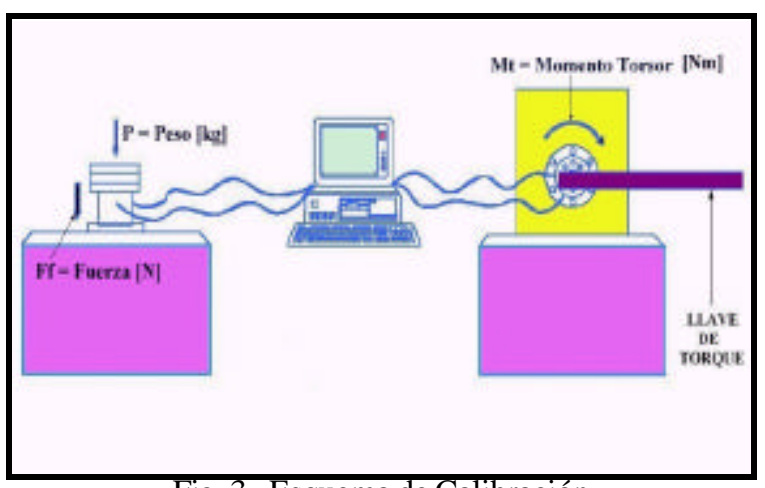

Fig. 3.- Esquema de Calibración

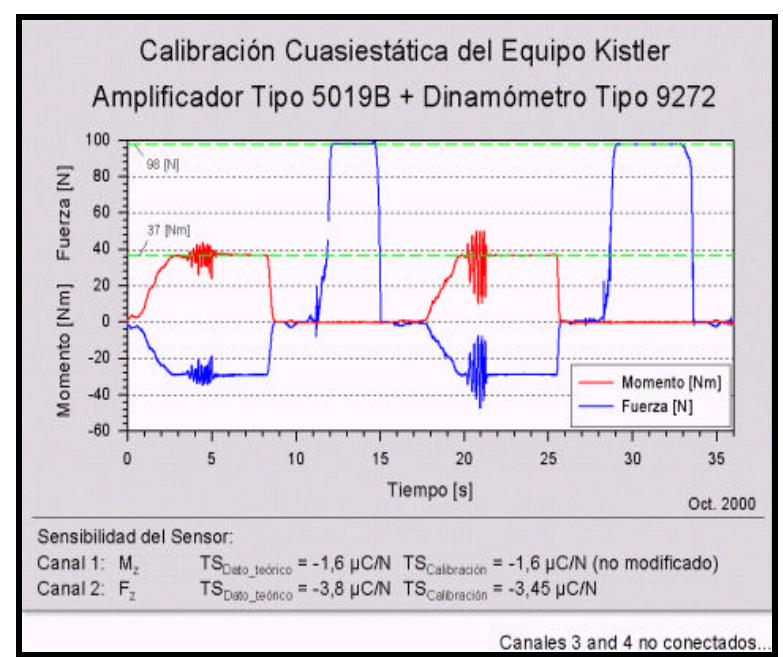

Fig. 4.- Resultados de la calibración
En la Fig. 5, se muestra un esquema del dispositivo para la fijación de los cuerpos de prueba. Este dispositivo se instala sobre el dinamómetro KISTLER. Este dispositivo fue fabricado en el Departamento de Mecánica de la Universidad de Tarapacá.

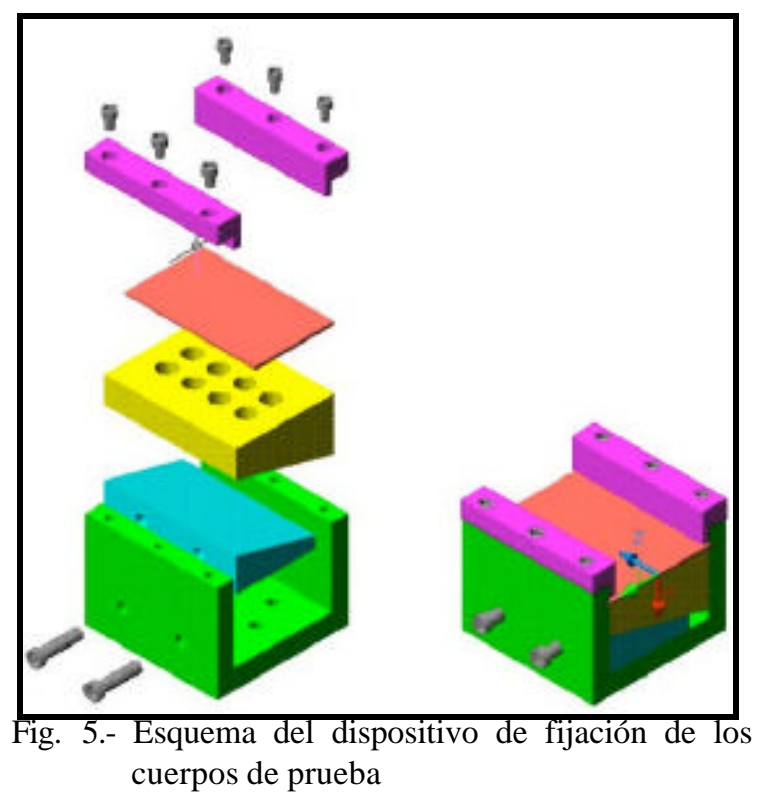

\section{MEDICIONES}

En todos los taladrados se utilizó una broca de carburo de tungsteno (WC) con un diámetro de 4,5 [mm].

La Fig. 6, muestra las curvas correspondientes a las fuerzas de avance, con una velocidad de 2000 [rpm] y un avance de 300 [mm/min.], en chapas de cobre de diferentes espesores.

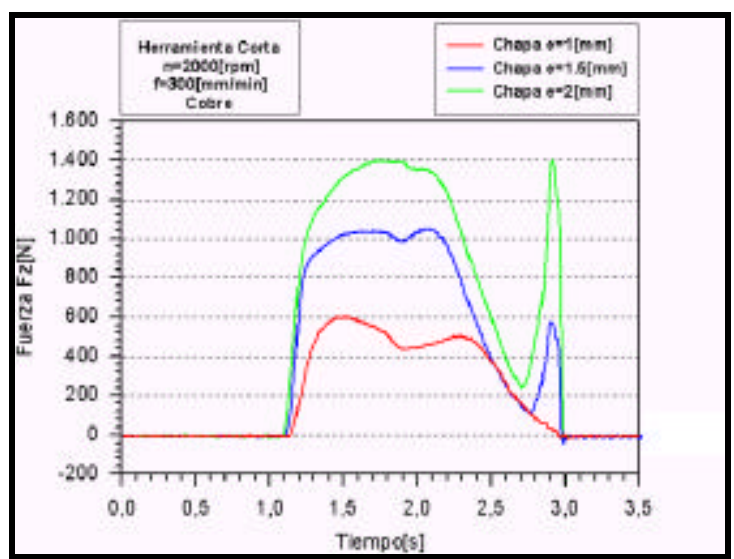

Fig. 6.- Fuerza de avance v/s Tiempo, para diferentes espesores 
En la Fig. 7, se muestra la variación de la fuerza de avance, para diferentes avances en $[\mathrm{mm} / \mathrm{min}]$, en las chapas de cobre, con una velocidad de rotación de 1000 [rpm]. Se observa un aumento creciente en la fuerza de avance, al aumentar el avance de la herramienta.

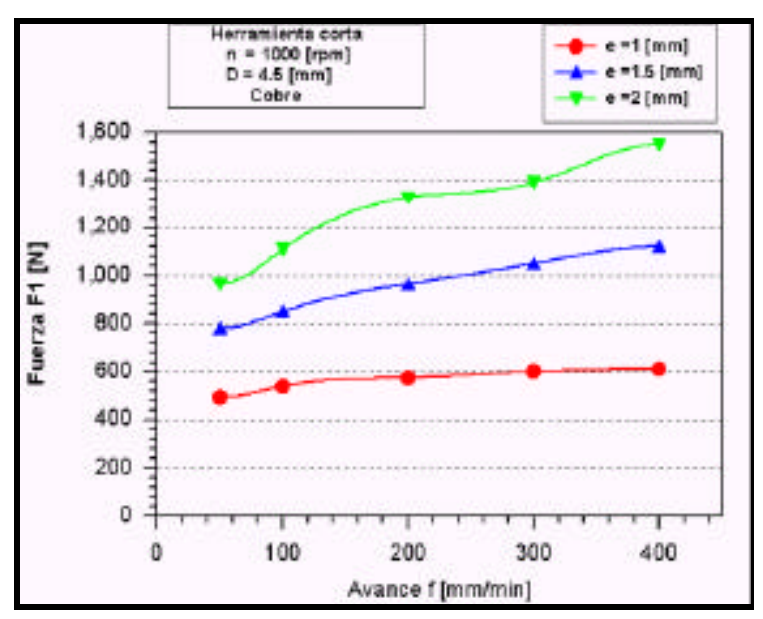

Fig. 7.- Fuerza de Avance v/s Avance

La Fig. 8, muestra la variación de la fuerza de avance para diferentes velocidades de rotación en chapas de cobre de distintos espesores. Se utilizó un avance de $\mathrm{f}=300 \quad[\mathrm{~mm} / \mathrm{min}$.$] . Se observa una disminución$ creciente en la fuerza de avance al aumentar la velocidad de rotación.

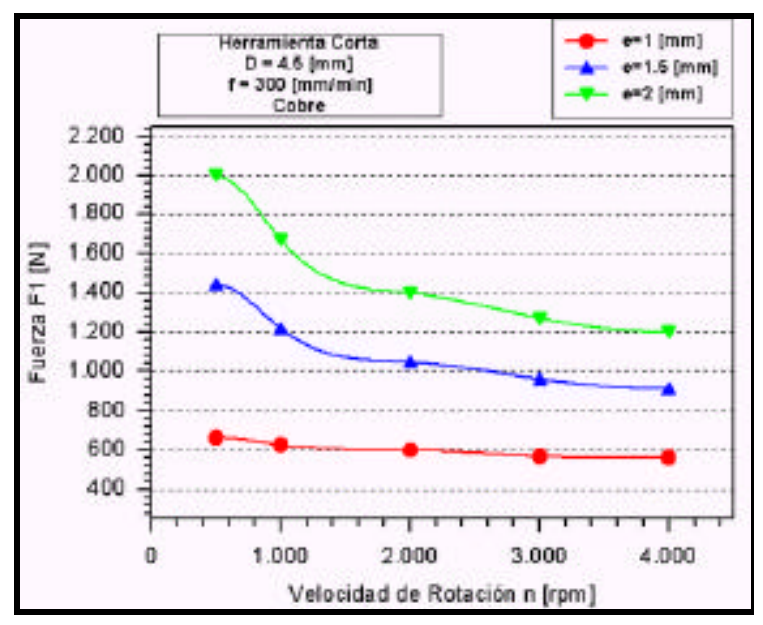

Fig. 8.- Fuerza de Avance v/s Velocidad de Rotación

La Fig. 9muestra la variación del momento torsor en las chapas de cobre. Los parámetros operacionales son: una velocidad de rotación de 2000 [rpm] y avance de 300 [mm/min.] Se observa un aumento del momento torsor al aumentar el espesor de la chapa de cobre.

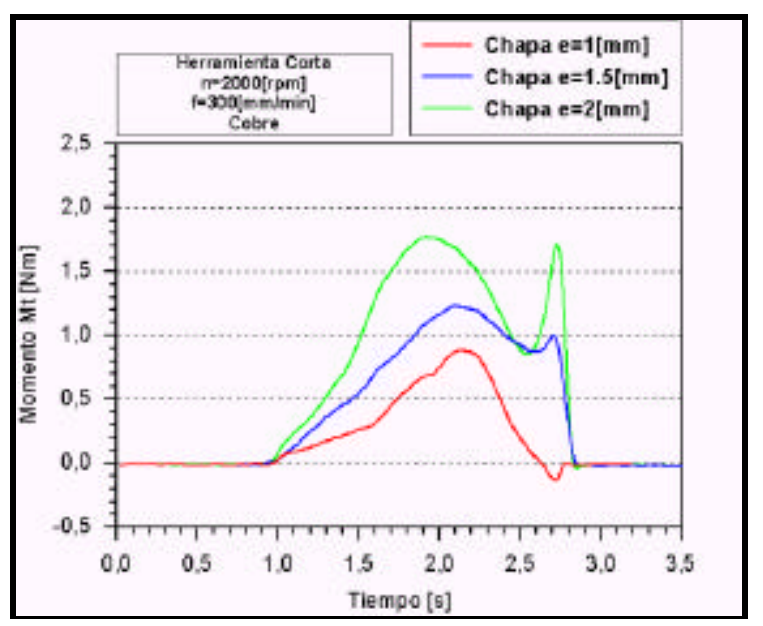

Fig. 9.- Momento Torsor v/s Tiempo, para diferentes espesores

La Fig. 10, muestra la variación del momento torsor para diferentes velocidades de rotación en chapas de cobre, para un avance $\mathrm{f}=300[\mathrm{~mm} / \mathrm{min}$.]. Se observa una disminución notoria en el momento torsor al aumentar la velocidad de rotación para la chapa de mayor espesor, en los otros espesores la disminución del momento no es tan acentuada.

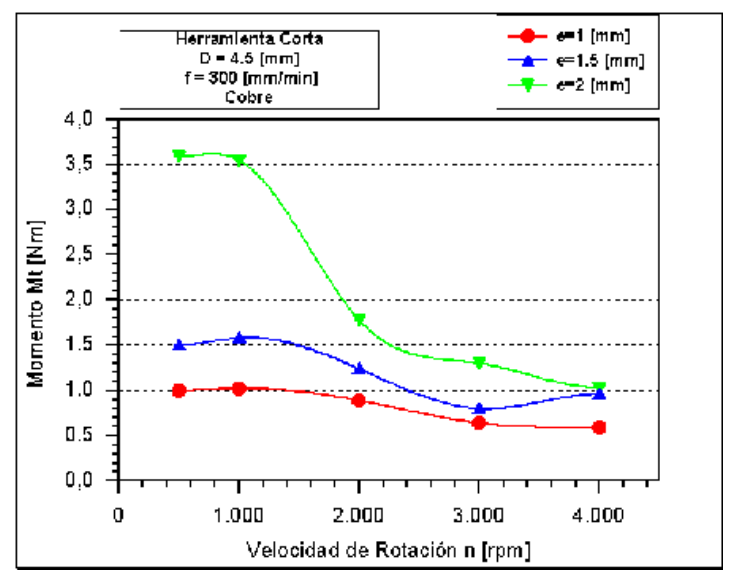

Fig. 10.- Momento Torsor v/s Velocidad de rotación

En la Fig. 11, al observar las fuerzas de avance con respecto al avance de la herramienta para cobre y latón, las fuerzas de avance son mayores para el latón. La razón de ello se debe a la mayor resistencia mecánica del latón con respecto a la resistencia del cobre. 
Juan Godoy R., Jorge Vergara D., Martín Quispe Y., Percy Oviedo O., Franz Tikal, Stefan Damm

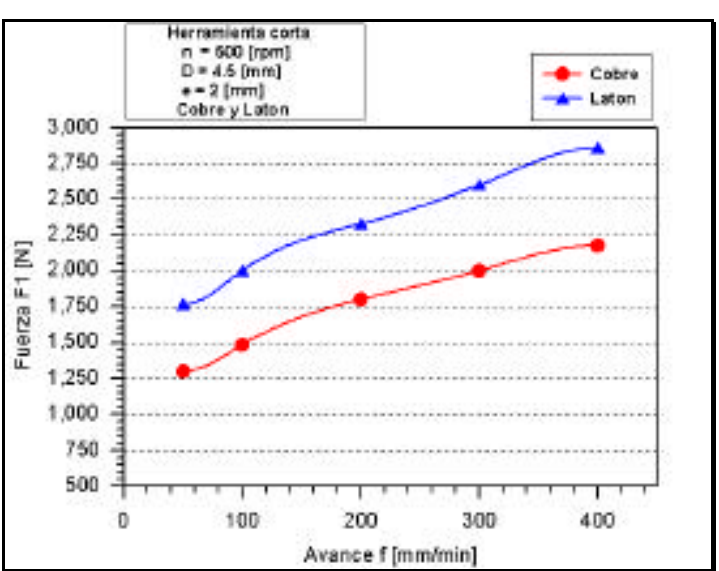

Fig. 11.- Fuerza de corte v/s Avance para Latón y Cobre

La Fig. 12, muestra el efecto del momento torsor con respecto al avance de la herramienta. Se observa nuevamente un momento torsor mayor para la chapa de latón.

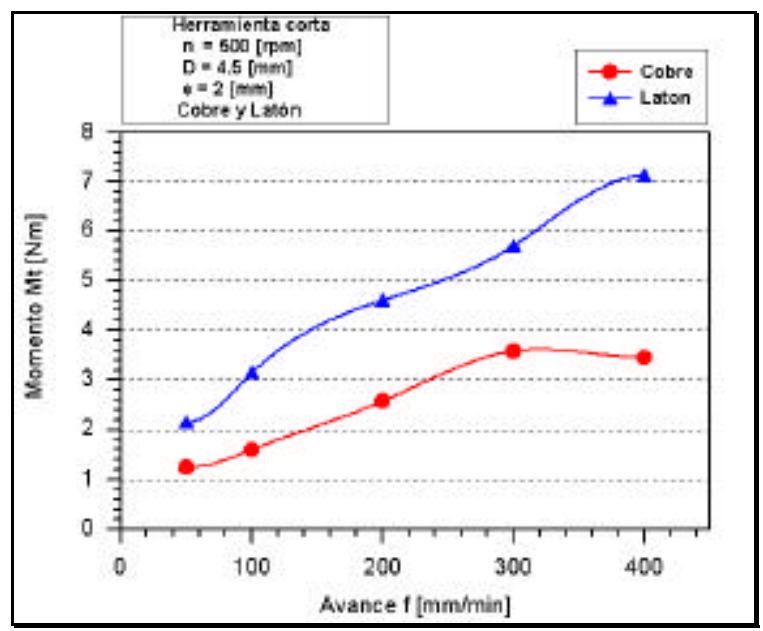

Fig. 12.- Momento Torsor máximo v/s Avance para Latón y Cobre

\section{CONCLUSIONES}

Se instaló en forma exitosa el dinamómetro KISTLER en el Centro de mecanizado vertical HAAS. Se calibró el equipo para la medición de las fuerzas de avance y momento. Se programó el PC con el software TESTPOINT, para la adquisición de los datos a través de la tarjeta DAS 82, con el software XACT. Se procesaron los datos.

Los diagramas de las fuerzas de avance muestran que al aumentar el avance de la herramienta y la velocidad de rotación, las fuerzas de avance aumentan en función del espesor, la mayor fuerza se presenta con la chapa de cobre de $2 \mathrm{~mm}$.

Los diagramas de momento torsor muestran que al aumentar la velocidad de rotación, se observa una disminución en el momento torsor, especialmente en la chapa de cobre de mayor espesor.

Los diagramas de fuerzas de avance y de momento torsor, son siempre mayores para las chapas de latón, cuando se las compara con chapas de cobre, para un mismo espesor.

\section{AGRADECIMIENTOS}

Se agradece a CONICYT - DAAD, proyecto ALECHILE, a la Universidad de Kassel de Alemania y Universidad de Tarapacá - Chile, por haber financiado el proyecto "Taladrado Térmico por Fluencia en Láminas delgadas.”

A la empresa FLOWDRILL B. V. de Holanda

\section{REFERENCIAS}

[1] T. Novosel; "Tapping without chips. American Machinist”, p.p. 103-105, Oct.1975.

[2] G. Kretschmer, Fließlochformer Von Blechdurchzügen. U. Siegen, Doctoral Tesis Maschinenbau - Universität Gesamthochschule Siegen 1980.

[3] J. C. Oliveira L.; Maestría "Taladrado por fluencia y Roscado por Conformación”, Universidad de Santa Catarina, Brasil, Florianopolis, abril 1994.

[4] W. Weingaertner.; Revista Máquinas e Metais, No 368, p.p. 85-97, septiembre 1996.

[5] J. C. Oliveira L.; Tesis de Doctorado "Una colaboración para la continuidad del desarrollo de la tecnología del Taladrado por Fluencia", Universidad de Kassel. Alemania, 1999. 